\title{
Tracing the evolution of knowledge structures
}

\author{
RICHARD K. WAGNER \\ Yale University, New Haven, Connecticut \\ MARC M. SEBRECHTS \\ Wesleyan University, Middletown, Connecticut \\ and \\ JOHN B. BLACK \\ Yale University, New Haven, Connecticut
}

\begin{abstract}
The goal of the research described here is to understand the evolution of knowledge structures as novices learn to use text editors. Two groups of individuals were trained to use one of two text editing systems: the IBM Displaywriter and UCSD-Pascal. Individuals rated the similarity among pairs of editing commands before and after training. Before training, subjects' similarity ratings are characterized by heavy reliance on natural language denotations of command names. After training, subjects' similarity ratings are explainable in terms of a small set of identifiable properties of underlying knowledge structures that are typical of experts' knowledge about a text editor. These properties reflect functional features of command actions, and the psychological salience of a property for a given individual is related to editing performance.
\end{abstract}

One of the most important issues in both basic and applied cognitive psychology is how the mental representations of novices change as they become expert at some cognitive skill. Recently, there has been an increasing amount of research on how the knowledge of experts differs from that of novices (e.g., Chi, Feltovich, \& Glaser, 1981; Ehrlich \& Soloway, 1983; Larkin, McDermott, Simon, \& Simon, 1980; Soloway, Ehrlich, Bonar, \& Greenspan, 1982). Although several theories of cognitive learning have been developed (e.g., Anderson, 1982; Rumelhart \& Norman, 1978; Schank, 1982) there has been little direct examination of how people's knowledge structures evolve as they learn a cognitive skill. In this paper, we present a study that illustrates one way to trace that evolution.

In particular, we report a study of the evolution of knowledge as novices learn to use text editors. Two groups of college undergraduates with no previous experience with text editors learned to use one of two text editing systems (IBM Displaywriter and UCSD-Pascal) over the course of a 1-week training program. We examined the evolution of knowledge about text editors through the use of several techniques, primarily modeling of changes in ratings of the similarities between pairs of editing commands as a function of training.

One way to gain insight into the evolution of people's knowledge about text editors is simply to ask them. We

This research was supported by National Science Foundation Grant 8217572 to $\mathrm{M}$. Sebrechts and by a grant from the IBM Corporation. The views expressed in this paper, however, are not necessarily endorsed by IBM. R. K. Wagner is now at Florida State University in Tallahassee. Send reprint requests to: Marc M. Sebrechts, Department of Psychology, Wesleyan University, Middletown, CT 06457. did, and found it generally to be a useful approach, but it was clear that users are unable to describe at least some aspects of their internalized representation of a text editing system. A second way to gain insight into the evolution of people's knowledge about text editors is to test them. We did, and learned quite a bit by watching individuals struggle fearlessly with and often succeed at a variety of editing tasks presented to them. But it takes a leap of inference to uncover underlying knowledge from observation and measurement of task performance. The subject of this report is a third way to gain insight into the evolution of knowledge, an approach that complements but does not replace either of the previously mentioned methods. In particular, we used hierarchical and overlapping clustering to model changes in perceived similarities among a subset of editing commands that resulted from learning to use text editors.

In order to understand changes in knowledge, we need to consider two aspects of performance on our similarity rating task: internal and external validation. Internal validation refers to breaking down task performance into underlying processes, parts, or subskills. External validation refers to demonstrating that the underlying units, and overall task performance itself, are related to performance on appropriate external tasks.

In the present study, internal validation was accomplished by modeling the observed similarities among editing commands. The principal technique used was overlapping clustering (Arabie \& Carroll, 1980; Shepard \& Arabie, 1979). Briefly, this technique groups editing commands into (possibly) overlapping command subsets, each of which corresponds to a discrete property or feature shared by a number of commands. Each such feature is also given a weight, which reflects the psychological 
salience of the underlying property for a given individual or group. External validation was then achieved by correlating measures of psychological salience with editing performance on related tasks.

\section{METHOD}

\section{Materials}

Twenty representative editing commands were selected from each of two word processing systems. Subjects learned the IBM Displaywriter system from either Training Book 1 (IBM, 1981) or a modified version of the training book that was primarily diagrammatic in format. Subjects learned the editor and filer portions of the UCSD-Pascal system (Softtech Microsystems, 1980) from one of two parallel manuals constructed for the experiment, one of which was primarily diagrammatic in format. For purposes of the present paper, we treat the two manuals for each system as equivalent.

Performance tests were presented that required subjects to create, revise, and restructure a document, as well as to perform a variety of editing tasks specified by the experimenters. Several standard tests of cognitive abilities and a test of typing ability were administered, and subjects responded to a questionnaire that asked about attitudes and knowledge about word processing systems and computers.

\section{Procedure}

Subjects participated in the training program for approximately $2 \mathrm{~h} /$ day for 5 consecutive days. Subjects gave similarity ratings for all possible nonidentical pairs of the 20 commands for their respective text editors before and after training. Command pairs were presented by computer, and subjects indicated their similarity rating (on a 7-point scale) by pressing the appropriate key on a standard keyboard. The order of command pairs and the order of commands within a command pair were randomized for each subject.

\section{Subjects}

Subjects in the Displaywriter group were 12 college students who had had no previous experience with text editors. Subjects in the UCSD-Pascal group were 11 college students or recent graduates who had had no previous experience with text editors.

\section{RESULTS}

\section{Internal Validity}

Two techniques were used to examine changes in knowledge structures with learning. First, hierarchical clustering was used to detect major changes in the patterns of similarities among editor commands after training. This technique groups commands according to their rated similarity. Items that are most similar are grouped together first, and subsequent clusters are formed by grouping items that are the next most similar. The second technique, overlapping clustering, was used for more precise examination of properties of the underlying knowledge structure.

The change in command similarity ratings as a result of training was striking and consistent in the hierarchical clustering results of both systems. Prior to training, natural language was the basis for perceiving commands to be similar. The early clusters formed for the UCSD-Pascal group (and hence, commands that were viewed as being most similar) included FIND and GET-the two commands that were perhaps the most closely related in natural language - and HEADER and MARGIN-two commands that refer to a typing environment. For the IBM Displaywriter group, early clusters included CODE and MESSAGE and also CANCEL and DELETE, both of which contain commands that share similar natural language meanings.

After training, the basis for perceiving commands to be similar shifted from natural language to function in the system. An early cluster for the UCSD-Pascal group included MARGIN and ADJUST, both of which are editor functions for changing the format of a page. Recall that, prior to training, MARGIN clustered with HEADER. FIND and GET, which were seen as highly similar prior to training, are now viewed as quite dissimilar. This reflects the fact that the FIND command functions in the editor to find text, and the GET command functions in the filer to get a stored document. The three major higher order clusters represent terminators, filer and printer commands, and editor commands. This underlying structure is highly similar to the system menu structure.

An early cluster for the IBM Displaywriter group after training contained CODE and CANCEL, two terms that are frequently used together when terminating a number of actions. Recall that, prior to training, CODE clustered with MESSAGE. The major higher order clusters represent requesting or printing, creating, screen formatting, code and cancel, and revising.

These results demonstrate that knowledge about the relationship among editor commands changes rather dramatically as a result of training, with functional connotations of editor commands gradually replacing the natural language denotations of command names.

Greater precision in specifying the nature of the properties of the underlying knowledge structure was obtained through the use of our second principal technique, overlapping clustering. Recall that overlapping clustering identifies properties shared by members of a command subset and provides a measure of the psychological salience of the property in the form of a weight for the subset.

Results for the UCSD-Pascal group are presented in Table 1 . Five command subsets accounted for $64 \%$ of the total variance in similarity ratings. This result was reliable $[\mathrm{F}(5,184)=65.4, \mathrm{p}<.001]$ and indicates that the properties captured by the subsets of commands account for the majority of the variance in the similarity ratings. The three most highly weighted subsets were readily interpretable. Commands in the first subset (ADJUST and MARGIN) share the property of being used to change the 
Table 1

Subsets and Weights for UCSD-Pascal Similarity Ratings After Training

\begin{tabular}{ccl}
\hline Subset & Weight & Commands Contained in Subset \\
\hline 1 & .80 & ADJUST, MARGIN \\
2 & .30 & $\begin{array}{l}\text { ADJUST, COPY, INSERT, EDIT, DELETE, } \\
\text { REPLACE, WRITE }\end{array}$ \\
3 & .24 & FILE, NEW, GET, SAVE, QUIT, UPDATE, PRINT \\
4 & .21 & $\begin{array}{l}\text { ADJUST, INSERT, QUIT, UPDATE, EDIT, DELETE, } \\
\text { ZAP, FIND, REPLACE, ESCAPE } \\
5\end{array}$ \\
& $\begin{array}{l}\text { COPY, FILE, NEW, GET, SAVE, MARGIN, INSERT, } \\
\text { LIST, UPDATE, EDIT, PRINT, FIND, REPLACE, } \\
\text { WRITE }\end{array}$ \\
\hline
\end{tabular}

format of a document. Commands in the second subset (ADJUST, COPY, INSERT, EDIT, DELETE, REPLACE, and WRITE) share the property of belonging to the editor proper, being used when creating and revising documents. Commands in the third subset (FILE, NEW, GET, SAVE, QUIT, UPDATE, and PRINT) share the property of being "machine" commands, being used when terminating editing sessions, printing files, and the like. The two least weighted subsets were not convincingly interpretable.

Results for the IBM Displaywriter system are presented in Table 2. Five command subsets accounted for $63 \%$ of the total variance in similarity ratings. This result was reliable $[\mathrm{F}(5,184)=62.7, \mathrm{p}<.001]$ and indicates that properties captured by the command subsets account for the majority of variance in similarity ratings. The two most highly weighted subsets were easily interpretable. Commands in the first subset (END, MESSAGE, PRINT, and REQUEST) share the property of being "machine" commands. Commands in the second subset (BACKSPACE, CANCEL, DELETE, FIND, REPLACE, and REVISE) share the property of being used in revising text. The properties underlying the remaining three subsets were less readily interpretable.

To summarize, when novices learn to use text editors, natural language meanings of command names recede and knowledge structures begin to emerge with identifiable underlying properites that reflect functional aspects of text editing. If this knowledge is important for performance, then the degree of evolution in that knowledge should be related to editing ability; that is, the structures should also have external validity.

\section{External Validity}

The analyses described in the previous section successfully accounted for much of the variance in subjects' similarity ratings. This section addresses the relationship between knowledge as represented in these ratings and actual editing performance by examining the fit of an "expert" model to individual performance data. Because of space limitations here, we will report these results only for the UCSD-Pascal group.

The expert model was devised by asking highly experienced users to describe the essential properties of the system. From among 20 properties, 5 were selected that were judged to be relatively important (e.g., "commands that make up the main editor," as opposed to "commands that rhyme"), and that accounted for at least some variance in group average similarity ratings. Commands that shared the first property-being part of the main editorwere EDIT, COPY, INSERT, MARGIN, DELETE, ADJUST, FIND, and REPLACE. Commands that shared the

Table 2

Subsets and Weights for IBM Displaywriter Similarity Ratings After Training

\begin{tabular}{ccl}
\hline Subset & Weight & Commands Contained in Subset \\
\hline 1 & .32 & END, MESSAGE, PRINT, REQUEST \\
2 & .31 & $\begin{array}{l}\text { BACKSPACE, CANCEL, DELETE, FIND, } \\
\text { REPLACE, REVISE }\end{array}$ \\
3 & .19 & $\begin{array}{l}\text { CREATE, ENTER, INSERT, MOVE, PRINT, } \\
\text { REPLACE, REQUEST, REVISE, SCREEN-FORMAT }\end{array}$ \\
4 & .16 & CENTER, FIND, INSERT, MOVE, REPLACE, RE- \\
& VISE, TAB, WORD-UNDERLINE \\
5 & BACKSPACE, CANCEL, CENTER, CODE, DELETE, \\
& INSERT, MOVE, RETURN, REVISE, SCREEN- \\
& FORMAT, TAB, WORD-UNDERLINE
\end{tabular}


second property - being part of the filer or "machine"were FILE, NEW, GET, and SAVE. Commands that shared the third property-being major terminators, or commands used when finished editing-were QUIT and ESCAPE. Commands that shared the fourth propertybeing text format modifiers-were ADJUST and MARGIN. Finally, commands that shared the fifth propertyused in search and replace operations-were FIND and REPLACE.

The expert model was fit to the similarity rating data individually for each subject. There were two questions of particular interest. First, do subjects who have more evolved models perform better? Second, is the psychological salience of the individual properties related to editing performance that should rely on awareness of those properties?

With regard to the first question, we assessed the development of an individual subject's knowledge by examining the goodness of fit of the expert model to the ratings given by the individual. The expert model accounted for a significant amount of variance for every subject in the UCSD-Pascal group. The percentage of variance accounted for ranged from $39 \%$ to $10 \%$, and the $F$ values ( $\mathrm{df}=5$ and 184 ) ranged from 23.5 to 4.09 , yielding a $\mathrm{p}<.01$ in every case. The correlation between the closeness of fit of an individual's data to the expert model (i.e., percentage of variance accounted for) and actual editing performance was 33 .

With regard to the second question, it was possible to examine the relationship between the psychological salience given a particular property and specific editing tasks for two of the expert properties--commands that make up the main editor (EDIT, COPY, INSERT, MARGIN, DELETE, ADJUST, FIND, and REPLACE) and commands that are used in search and replace operations (FIND and REPLACE). The correlation between the psychological salience given to the editor property (as measured by the weight given that subset) and the average number of attempts needed to make an editing modification on the most reliable of the editing tasks was -.44. This means that the greater the salience of the main-editor property, the more efficient the individual was at modifying text.

The correlation between the psychological salience given to the property of commands that are used in search and replace operations and the average number of attempts needed to complete a find and replace operation during actual editing performance was -.42 . This again confirms the link between user knowledge as represented by similarity ratings and actual editing performance.

\section{CONCLUSIONS}

We have argued for applying the criteria of internal and external validity to techniques for examining the evolution of knowledge structures as a result of learning to use a text editor. Methods for demonstrating internal and external validity of knowledge structures were described and used for validation of one method of examining the evolution of knowledge structures-namely, the method of analyzing changes in patterns of similarity ratings for editing commands due to learning to use a text editor. The major substantive findings were that novices depend heavily on the natural language meanings of command names during initial learning, but that, with experience, they begin to acquire the functional knowledge of editing commands that is possessed by experts. In addition, the more the structures revealed by the similarity ratings resembled the structures of experts, the better the novices performed on the system.

The methodology we have described and applied in this specific case is general in two ways. First, it can be applied to types of data other than similarity ratings. For example, similar methods could be applied to analyze performance errors (e.g., confusions between commands). Second, the methodology applies equally well to studying the acquisition of programming languages as it does to learning to use a command language like a text editor. Furthermore, since the techniques help to identify potential conceptual difficulties, they can be used to improve training and design by isolating the sources of user problems.

\section{REFERENCES}

Anderson, J. R. (1982). Acquisition of cognitive skill. Psychological Review, 89, 369-406.

ARABIE, P., \& Carroll, J. (1980). MAPCLUS: A mathematical programming approach to fitting the ADCLUS model. Psychometrika, 45, 211-235.

Chi, M. T. H., Feltovich, P. J., \& Glaser, R. (1981). Categorization and representation of physics problems by experts and novices. Cognitive Science, 5, 121-152.

EHRLICH, K., \&oloway, E. (1983). An empirical investigation of the tacit plan knowledge in programming. In J. Thomas \& $\mathbf{M}$. Schneider (Eds.), Human factors in computer systems. Norwood, $\mathrm{NJ}$ : Ablex.

IBM. (1981). IBM Displaywriter system operator training book 1Basjc topics. Austin, TX: Author.

Larkin, J. H., MCDermotr, J., Simon, D. P., Simon, H. (1980) Expert and novice performance in solving physics problems. Science, 208, 1335-1342.

Rumelhart, D. E., \& Norman, D. A. (1978). Accretion, tuning and restructuring: Three modes of learning. In J. W. Cotton \& R. Klatzky (Eds.), Semantic factors in cognition. Hillsdale, NJ: Erlbaum.

SCHANK, R. C. (1982). Dynamic memory: A theory of reminding and learning in computers and people. Cambridge, England: Cambridge University Press.

ShEPARD, R., \& ARABIE, P. (1979). Additive clustering: Representations of similarities as combinations of discrete overlapping properties. Psychological Review, 86, 87-123.

SOFTTECH MICROSYSTEMS. (1980). UCSD PASCAL user's manual. San Diego, CA: Author.

SOlOWAy, E., EhrLich, K., Bonar, J., \& Greenspan, J. (1982). What do novices know about programming? In A. Badre \& B. Shneiderman (Eds.), Directions in human computer interaction. Norwood, NJ: Ablex. 